\title{
TIPE2, a negative regulator of TLR signaling, regulates p27 through IRF4-induced signaling
}

\author{
YANPING PENG $^{1 *}$, QIAN ZHAO ${ }^{1 *}$, HANYU ZHANG $^{1 *}$, BO HAN $^{1}$, SUXIA LIU $^{1}$, \\ MINGYONG HAN ${ }^{1,2}$ and SHILI LIU ${ }^{1}$ \\ ${ }^{1}$ School of Medicine, Shandong University; ${ }^{2}$ Cancer Therapy and Research Center, \\ Shandong Provincial Hospital, Shandong University, Jinan, Shandong 250012, P.R. China
}

Received October 16, 2015; Accepted December 31, 2015

DOI: $10.3892 /$ or.2016.4562

\begin{abstract}
Targeted inhibition of specific toll-like receptor (TLR) pathways may provide an effective strategy to prevent the development of selected gastric malignancies. Tumor necrosis factor (TNF)- $\alpha$-induced protein 8-like-2 (TIPE2) was identified as a novel negative regulator of TLR signaling. Our previous study identified TIPE2 as an inhibitor of gastric cancer cell growth; it promotes $\mathrm{p} 27$ expression, which leads to restored control of the cell cycle and cell division. However, the molecular mechanism by which TIPE2 regulates p27 remains unclear. In the present study, we examined the expression patterns of TIPE2 in serial clinical gastritis tissues as well as gastric cancer, and found a negative correlation between TIPE2 expression and progression of gastritis to gastric cancer. This negative correlation verified the role of TIPE2 in preventing the occurrence and development of gastric cancer, suggesting that TIPE2 may be a potential biomarker for gastric cancer progression. To determine the mechanism employed by TIPE2 in gastric cell carcinogenesis, a TIPE2expressing plasmid was introduced into gastric cell lines, and microarray and western blot analysis revealed that TIPE2 selectively upregulates the expression of interferon regulatory factor 4 (IRF4). Variations in IRF4 expression were additionally verified in knockout mice. Next, the effect of IRF4 on p27 expression was tested by an IRF4 siRNA interference assay. Finally, we explored the signaling pathways used by TIPE2 to regulate IRF4. An experiment using pathway inhibitors and a nuclear factor $\kappa$-light-chain enhancer of activated $B$ cells

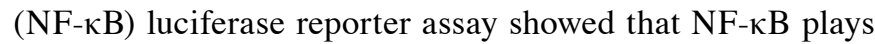
a crucial role in regulating IRF4 expression. Our data provide evidence that TIPE2, a potential biomarker for gastric cancer
\end{abstract}

Correspondence to: Dr Shili Liu, Department of Medical Microbiology, School of Medicine, Shandong University, Jinan, Shandong 250012, P.R. China

E-mail: liushili@sdu.edu.cn

${ }^{*}$ Contributed equally

Key words: tumor necrosis factor (TNF)- $\alpha$-induced protein 8-like-2, interferon regulatory factor 4 , toll-like receptor, p27, NF- $\kappa B$ progression, stimulates an IRF4-associated signaling cascade that promotes $\mathrm{p} 27$ expression and controls cell growth. To the best of our knowledge, this is the first study to demonstrate that IRF4 acts as an inhibitor of epithelial cell proliferation and mediates the expression of TIPE2, a negative regulator of TLR signaling, to control cell growth.

\section{Introduction}

Gastric cancer (GC) accounts for $\sim 10 \%$ of all invasive cancers worldwide $(1,2)$ and is the second leading cause of cancer-related death worldwide (3), resulting in an urgent medical need to develop more efficient diagnosis methods and effective treatments. While the pathogenesis of the disease is not completely understood, epidemiological studies suggest that chronic inflammation plays a significant role in the development of gastric malignancies (4). In superficial gastritis (SG), inflammatory changes only affect the superficial epithelium gastric pit region and related lamina propria, but the changes occur in the atrophic gastric glands (5). Given that the recognition of bacterial and/or viral products by toll-like receptor (TLR)-expressing cells induces the activation of nuclear factor $\kappa$-light-chain-enhancer of activated B cells $(\mathrm{NF}-\mathrm{kB})$ and triggers an inflammatory response associated with tumor promotion (6), the targeted inhibition of specific TLR pathways may provide an effective strategy for preventing the development of selected gastric malignancies. To date, several negative regulators of TLR signaling pathways have been identified and characterized (7), among which tumor necrosis factor (TNF)- $\alpha$-induced protein 8-like-2 (TIPE2) and interferon regulatory factor 4 (IRF4) exert their activity via dissociation of TLR adaptor complexes (8).

TIPE2, a member of the tumor necrosis factor- $\alpha$-induced protein-8 (TNFAIP8) family, was recently identified as a novel negative regulator of the immune system that independently maintains immune homeostasis (9). In vitro experiments demonstrated that TIPE2 knockout cells showed hyperresponsiveness to TLR and T cell receptor (TCR) activation (9). In addition to lymphoid tissue, TIPE2 is expressed in the nervous, digestive, urinary, respiratory and reproductive systems (10-14), suggestive of roles other than immune regulation. Our previous results demonstrated that TIPE2 regulates the proliferation of gastric cells (15). Colony-forming assays 
showed that restoration of TIPE2 expression in gastric cells significantly suppressed cell proliferation. Flow cytometric analysis showed that the number of cells in the $S$ phase of the cell cycle was reduced concomitant with TIPE 2 expression. In addition, TIPE2 selectively upregulated p27 expression, which controls cell growth; however, the molecular mechanism by which TIPE2 regulates p27 remains unknown.

IRF4 also participates in the negative regulation of TLR signaling as well as the regulation of inflammation and carcinogenesis. Negishi et al (16) found that IRF4 forms a complex with MyD88, functioning not only as a transcription factor in lymphocyte differentiation but also as a negative regulator of TLR signaling. IRF4 is also expressed in macrophages, lens cells and melanocytes, suggestive of its involvement in the regulation of cells other than lymphocytes. Studies by Pathak et al demonstrated that reconstitution of IRF4 expression restored p27 in leukemic cells and inhibited cell proliferation in vivo (17). Moreover, Fanzo et al (18) showed that stable IRF4 expression in a human lymphoid cell line that normally lacks IRF4 significantly enhanced the apoptotic response in Fas receptor engagement. However, the effects of IRF4 on p27 expression and growth modulation as well as upstream molecules of IRF4 in epithelial cells are yet to be explored.

In the present study, initial examination of TIPE2 expression patterns in superficial gastritis (SG, 22 patients), atrophic gastritis (AG, 30 patients), atypical hyperplasia (AH, 24 patients) and gastric cancer (GC, 34 patients) tissues revealed a negative correlation between TIPE2 expression and tumor development and malignant progression. This negative correlation demonstrates the role of TIPE2 in preventing the occurrence and development of GC, and suggests that TIPE2 may be a potential biomarker for GC progression. To elucidate downstream molecular targets, a TIPE2-expressing plasmid was introduced into the AGS gastric epithelial cell line. Microarray analysis of signal transduction data revealed that TIPE2-induced IRF4 expression in AGS cells, and in turn, enhanced p27 expression and suppressed cell proliferation. To the best of our knowledge, this is the first study to demonstrate that TIPE2 expression is mediated by another negative TLR signaling regulator, IRF4, to modulate cell growth, and IRF4 functions as an inhibitor of epithelial cell proliferation.

\section{Materials and methods}

Patient samples. Newly obtained endoscopic biopsy specimens from $22 \mathrm{SG}, 30 \mathrm{AG}, 24 \mathrm{AH}$ and $34 \mathrm{GC}$ tissues were obtained from The Second Hospital of Shandong University (Jinan, Shandong, China). All studies were reviewed and approved by the Ethics Committee of Shandong University. All the patients gave their informed consent prior to their inclusion in the study. The tissues were kept at $-80^{\circ} \mathrm{C}$ and used for subsequent analysis.

Immunohistochemistry for TIPE2 protein expression. Immunohistochemical analysis was performed according to a previous study (12). Briefly, sections were thawed and fixed in acetone for $10 \mathrm{~min}$ at $20^{\circ} \mathrm{C}$, then rehydrated in $0.1 \mathrm{M}$ phosphate-buffered saline (PBS) for $5 \mathrm{~min}$. Next, the sections were treated by blocking goat serum for $10 \mathrm{~min}$, rabbit anti-TIPE2 polyclonal antibody (dilution 1:50; Boster Co. Ltd., Wuhan, China) for $60 \mathrm{~min}$, and then with peroxidase-labeled goat antirabbit IgG antibody (Maixin Bio, Fuzhou, China) for $15 \mathrm{~min}$, respectively, at room temperature. DAB peroxidase substrate kit (Maixin Bio) was used to quantify the peroxidase. Finally the sections were counter-stained with hematoxylin.

According to both staining intensity and percentage of positive cells (H-score) (19), all slides were scored as: staining intensity, 0 , no staining; 1 , weak staining; 2 , moderate staining; 3, strong staining; percentage of positive cells, $0,<1 \% ; 1,1-10 \% ; 2,10-25 \% ; 3,25-50 \%$; and $4,>50 \%$. The staining intensity multiplied by the percentage of positive cells in each slide produced a final score of TIPE2 expression and was graded as: 0 , final score $=0 ; 1+$, final score $=1-3 ; 2+$, final score $=4-6 ; 3+$, final score $=7-9 ;$ and $4+$, final score $=10-12$.

Cell culture and transfection. Gastric adenocarcinoma cell line AGS and BGC-823 were maintained in our laboratory. AGS cells were cultured in Ham's F-12 medium (HyClone, Logan, UT, USA) containing $10 \%$ fetal calf serum (FCS) and $1 \%$ penicillin-streptomycin. BGC-823 cells were cultured in RPMI-1640 medium (Life Technologies, Foster City, CA, USA) supplemented with $10 \%$ FCS (Tianhang Co. Ltd., Hangzhou, China) and $1 \%$ penicillin-streptomycin. The full-length human TIPE2 cDNA expression plasmid pRK5-tipe2 and control plasmid pRK5-mock were kindly provided by Professor Youhai Chen (University of Pennsylvania, USA) and were previously described (12). FuGENEs HD Transfection Reagent (Roche Applied Science, Basel, Switzerland) was used for transfection. All transfections were performed according to the manufacturer's instructions.

RNA extraction and quantitative real-time PCR. Total cellular RNA was extracted with TRIzol (Life Technologies) according to the protocol provided by the manufacturer. First-strand cDNA was synthesized from $1 \mu \mathrm{g}$ total cellular or tissue RNA using the RevertAid ${ }^{\mathrm{TM}}$ First Strand cDNA Synthesis kit (Thermo Fisher Scientific, Waltham, MA, USA) with random primers. Then cDNA was amplified for quantitative real-time PCR, the specific primers used were as follows: for TIPE2 forward primer, 5'-CTGAGTAAGATGGCGGGTCG-3' and reverse primer, 5'-TCTGGCGAAAGCGGGTAG-3'; for $\beta$-actin forward primer, 5'-AGTTGCGTTACACCCTTTCT TG-3' and reverse primer, 5'-CACCTTCACCGTTCCAGT TTT-3'; for p27 forward primer, 5'-GGTTAGCGGAGCAAT GCG-3' and reverse primer, 5'-TCCACAGAACCGGCATT TG-3'; for human IRF4 forward primer, 5'-AAAGGAAAGTT CCGAGAAGG-3' and reverse primer, 5'-CGAAGGGTAAGG CGTTGT-3'; for mouse IRF4 forward primer, 5'-CTCTTCAA GGCTTGGGCATT-3' and reverse primer, 5'-TGCTCCTTTT TTGGCTCCCT-3'. The real-time PCR reactions were performed at: $95^{\circ} \mathrm{C}, 10 \mathrm{sec}$ (denaturation); $55^{\circ} \mathrm{C}, 30 \mathrm{sec}$ (annealing); $72^{\circ} \mathrm{C}, 30 \mathrm{sec}$ (extension) for 35 cycles. The real-time PCR reactions were performed on the ABI 7000 Fast Real-Time PCR system with SYBR Premix Ex Taq ${ }^{\mathrm{TM}}$ according to the procedures.

Western blot analysis. Western blot analysis was performed as previously described (20). Briefly, cell lysates (20 $\mu \mathrm{g} / \mathrm{lane})$ were separated on $10 \%$ SDS polyacrylamide gel and were then 
transferred to a poly(vinylidene fluoride) membrane. TIPE2 and IRF4 protein was detected by a rabbit polyclonal $\mathrm{IgG}$ (Boster Co. Ltd.) and visualized by the enhanced chemiluminescence system (Amersham, Arlington Heights, IL, USA). The density of the bands was quantitated using the NIH image software package (version 1.61). The intensity of TIPE2 and IRF4 expression was judged by the ratio of their expression in experiment groups to their corresponding expression in control groups and a ratio of $>1.0$ was considered to be an indication of overexpression.

Colony formation assay. Gastric cell line AGS cultured in a 6-well plate ( $2 \times 10^{5} /$ well) was transfected with pRK5-tipe2 and its control plasmid pRK5-mock using the FuGENEs HD transfection reagent (Roche Applied Science). After a certain time of growth the cells were digested with trypsin and counted, 300 cells were transferred to a new well of a 6-well plate and medium containing $10 \%$ fetal bovine serum (FBS) serum was added to make up the volume of $3 \mathrm{ml}$. After a week's growth at $37^{\circ} \mathrm{C}$, the formation of cell clones could be visually seen. After washing 3 times with PBS buffer, the cells were fixed for $10 \mathrm{~min}$ with $1 \mathrm{ml}$ of methanol in each well at room temperature. Then, $1 \mathrm{ml}$ diluted Giemsa dye was added to each well and incubated at room temperature for $20-25 \mathrm{~min}$. Finally, the wells were washed with PBS until no residual background Giemsa dye was observed and the 6-well plate was scanned for colony counting and analysis.

Microarray analysis. The microarray chip consisted of 27,326 probes for different human cDNAs (Agilent Technologies, Wilmington, DE, USA), in which house-keeping gene glyceraldehyde-3-phosphate dehydrogenase (GAPDH) served as internal control. The cDNAs from pRK5-tipe2 transfected AGS cells were labeled with Cy3, and the cDNAs from the control pRK5-mock transfected AGS cells were labeled with Cy5. The labeled cDNAs were hybridized with microarray chip under standard conditions according to the manufacturer's instructions. The data were analyzed by Molecule annotation system 3.0.

siRNA interference. Chemical modified stealth siRNA targeting IRF4 and control siRNA were from RiboBio Co., Ltd. (Guangzhou, Guangdong, China). The sequence for IRF4 siRNA was 5'-UGGAGCGUGAGAGUCAAAG-3'. Cells were transfected with siRNA by the Lipofectamine 2000 method (Life Technologies).

Statistical and data analyses. Data are expressed as mean \pm standard deviation (SD). Differences between three groups were compared using the Student's t-tests. All experiments were repeated at least 3 times and $p<0.05$ was considered statistically significant.

\section{Results}

TIPE2 expression is negatively correlated with the progression of gastritis to cancer. To examine the expression pattern of TIPE2 in serial clinical tissue with the development of GC from gastritis, immunohistochemical analysis of TIPE2 protein was performed in gastric tissues collected from patients with
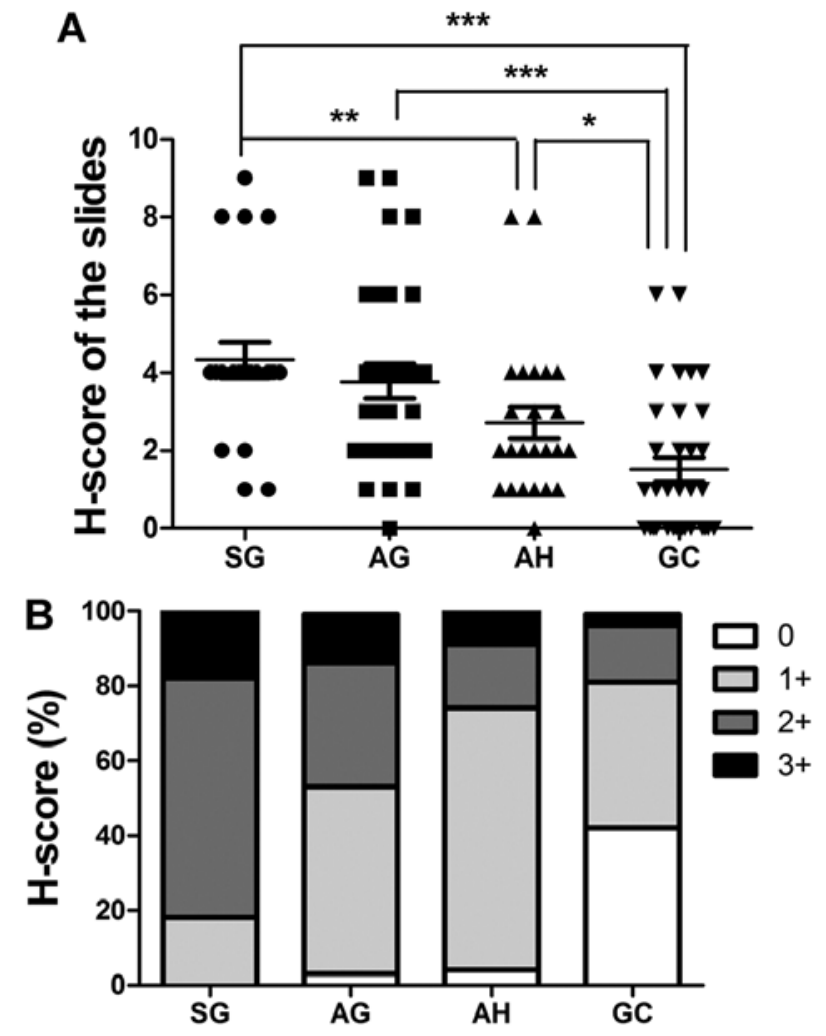

Figure 1. Expression levels of TIPE2 in gastric cells. Immunohistochemical scores of TIPE2 were based on both staining intensity and percentage of positive cells (H-score) in all slides. Staining was scored as follows: staining intensity, 0 , no staining; 1 , weak staining; 2 , moderate staining; 3 , strong staining. The percentage of positive cells was scored as follows: $0,<1 \%$; $1,1-10 \% ; 2,10-25 \% ; 3,25-50 \%$; and $4,>50 \%$. Staining intensity was multiplied by the percentage of positive cells for each slide to produce a final $\mathrm{H}$-score of TIPE2 expression (A). (B) The H-scores of (A) were graded: $0, \mathrm{H}$-score $=0 ; 1+, \mathrm{H}$-score $=1-3 ; 2+, \mathrm{H}$-score $=4-6 ; 3+, \mathrm{H}$-score $=7-9 ;$ and $4+$, H-score $=10-12$. Rabbit anti-TIPE2 polyclonal antibody (1:50 dilution) was used for immunohistochemical analysis.

SG (22 patients), AG (30 patients), AH (24 patients) and GC (34 patients). Each of these four diseases represents a stage of GC progression. Immunohistochemistry scores were based on both staining intensity and percentage of positive cells in all of the slides (H-score). As shown in Fig. 1A and B, TIPE2 expression in SG tissues significantly differed from expression in $\mathrm{AH}(\mathrm{p}=0.0118)$ and $\mathrm{GC}(\mathrm{p}<0.0001)$ tissues. $\mathrm{AG}$ and $\mathrm{AH}$ tissues also had verified levels of TIPE2 compared to GC tissues $(\mathrm{p}<0.0001$ and $\mathrm{p}=0.0203$, respectively). The percentage of highly scored slides (2+ and $3+$ ) decreased in the following order: SG, AG, AH and GC, whereas low scoring slides (total score $=0$ ) increased in this order. These variations in TIPE2 expression patterns with disease progression clearly demonstrate that its expression is suppressed in deteriorating gastritis and cancer tissues. Our finding, that TIPE2 expression is significantly negatively correlated with the progression of gastritis to GC, further supports the theory that TIPE2 is a molecular bridge from inflammation to cancer. Therefore, TIPE2 plays a role in the prevention of GC progression and may be a potential biomarker for GC progression.

TIPE2 upregulates IRF4 expression in gastric epithelial cells. To simulate TIPE2 expression in gastric cell lines and establish 


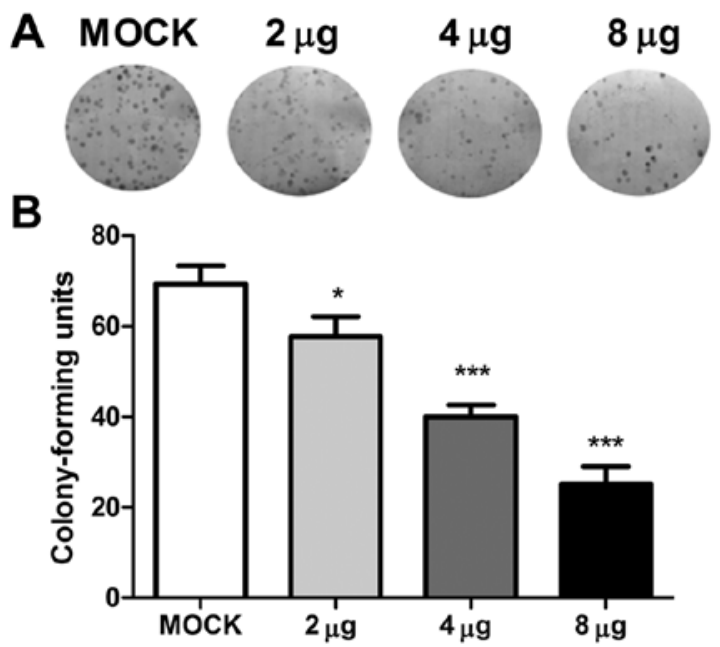

Amount of transfected pRK5-tipe2

Figure 2. TIPE2 expression significantly suppresses proliferation of AGS cells in a dose-dependent manner. (A) Colonies formed on agar; (B) quantification of colonies on agar, $\mathrm{p}<0.01$. Colony formation assays were performed as described in Materials and methods. Experiments were performed in triplicate with similar results.
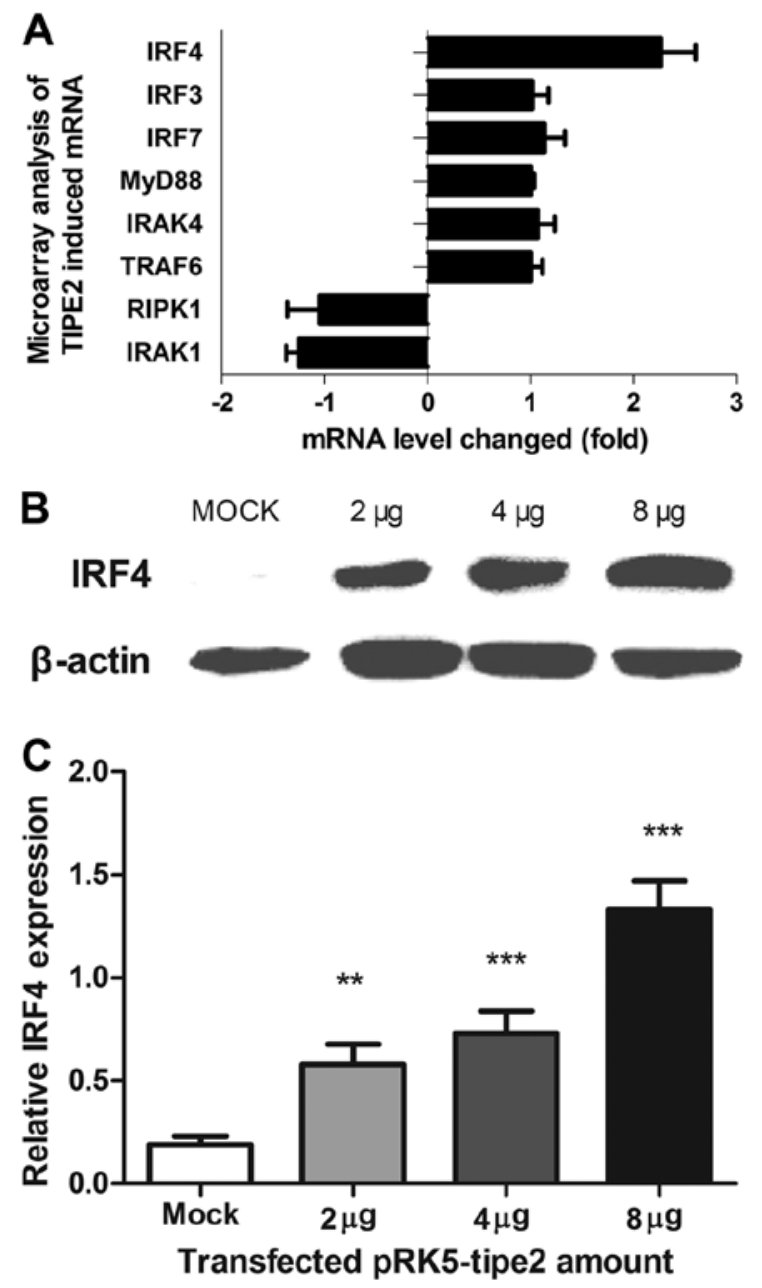

Figure 3. TIPE2 upregulates IRF4 expression in human and mouse cells. (A) Microarray analysis of the genes related to TLRs pathway upon TIPE2 expression in AGS cells, IRF4 was demonstrated with $>2$-fold increase in mRNA, as verified by (B) western blotting and (C) RT-qPCR. Microarray was performed in duplicate; western blotting and RT-qPCR in triplicate with similar results.

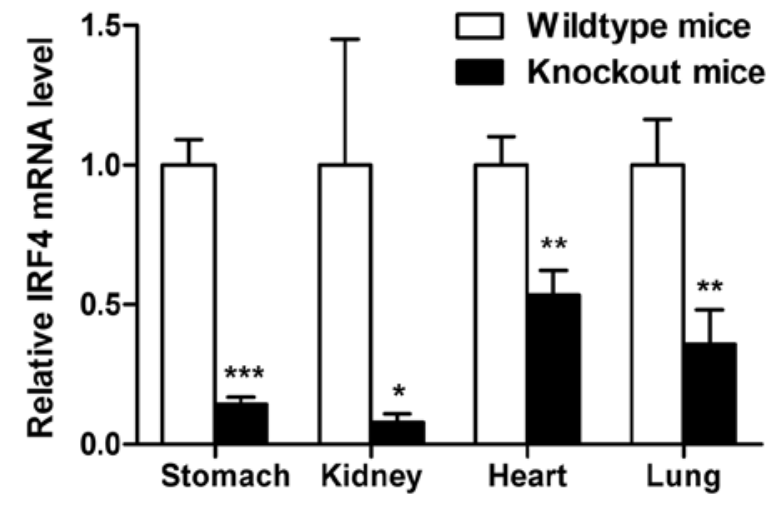

Figure 4. Examination of TIPE2-induced IRF4 expression in TIPE2 knockout mice disclosed downregulation of IRF4 in the stomach, kidney, heart and lung. RT-qPCR were performed as described in Materials and methods. Experiments were performed in triplicate.

its function in carcinogenesis, we transfected AGS cells with the TIPE2 expression plasmid, pRK5-tipe2; our results proved that the transfection efficiency was high under transfection with up to $8 \mu \mathrm{g}$ plasmid. As shown in Fig. $2 \mathrm{~A}$ and B, cells with restored TIPE2 expression had a significantly decreased colony forming capability; thus, the number and colony size were reduced compared to control cells transfected with mock plasmid. This growth inhibition effect was also observed in the BGC-823 gastric cell line.

To determine the molecular agents involved in TIPE2induced growth inhibition, cDNA microarray assays were conducted to analyze changes in gene levels upon TIPE2 expression. IRF4 was among the top upregulated genes with a $>2$-fold increase in expression, as confirmed by western blot analysis and quantitative PCR (qPCR) (Fig. 3A-C). Moreover, IRF4 mRNA and protein levels increased in a dose-dependent manner upon transfection of the pRK5-tipe2 plasmid in AGS cells, consistent with the TIPE2 expression pattern. TIPE2induced IRF4 expression in TIPE2 knockout mice led to downregulation of IRF4 in the stomach (Fig. 4). Similar results were obtained from other organs of TIPE2 knockout mice.

Next, we determined whether AGS cell growth and p27 expression level are modulated, concomitant with TIPE2 administration and IRF4 interference. As shown in Fig. 5A and B, cells transfected with the TIPE2 expression plasmid and IRF4 siRNA showed restored colony forming capacity and colony size. Knockdown of IRF4 protein was verified in specific cells (Fig. 5C). Subsequently, expression of p27 that TIPE2 selectively upregulated and takes control of cell growth was investigated. The p27 mRNA level was clearly decreased upon transfection of IRF4 siRNA (Fig. 5D). These results suggest that IRF4 plays a role in p27 regulation, and as such, is a critical mediator of TIPE2-induced growth inhibition.

Pathways employed by TIPE2 to regulate IRF4 expression. Next, we explored the signaling pathways used by TIPE2 to regulate IRF4, which plays a pivotal role in cell proliferation. The small molecule inhibitors SB203580 (p38 inhibitor, $10 \mu \mathrm{M}$ ), PD98059 (MEK inhibitor, $25 \mu \mathrm{M}$ ), SP600125 (JNK inhibitor, $10 \mu \mathrm{M}$ ), LY294002 (PI3K inhibitor, $40 \mu \mathrm{M}$ ), curcumin (AP-1 

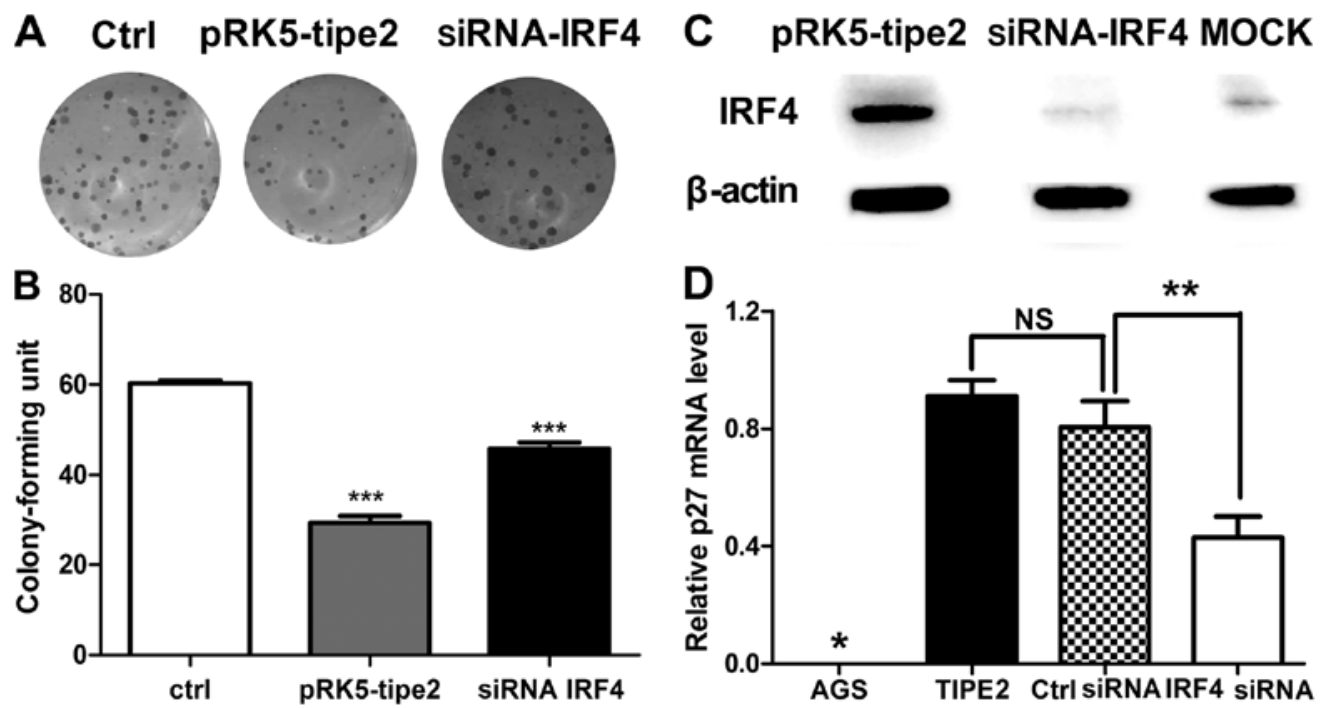

Figure 5. Cells transfected with TIPE2 expression plasmid and siRNA showed restored colony forming capacity and colony size, as well as p27 expression. (A) Cells transfected with TIPE2 expression plasmid and IRF4 siRNA showed restored colony forming capacity and colony size. (B) Quantification of (A). (C) Knockdown of IRF4 protein was verified in AGS cells. (D) Cells transfected with TIPE2 expression plasmid and IRF4 siRNA abolished p27 expression. siRNA interference, colony forming assay and western blotting were performed as described in Materials and methods. Experiments were performed in triplicate with similar results.

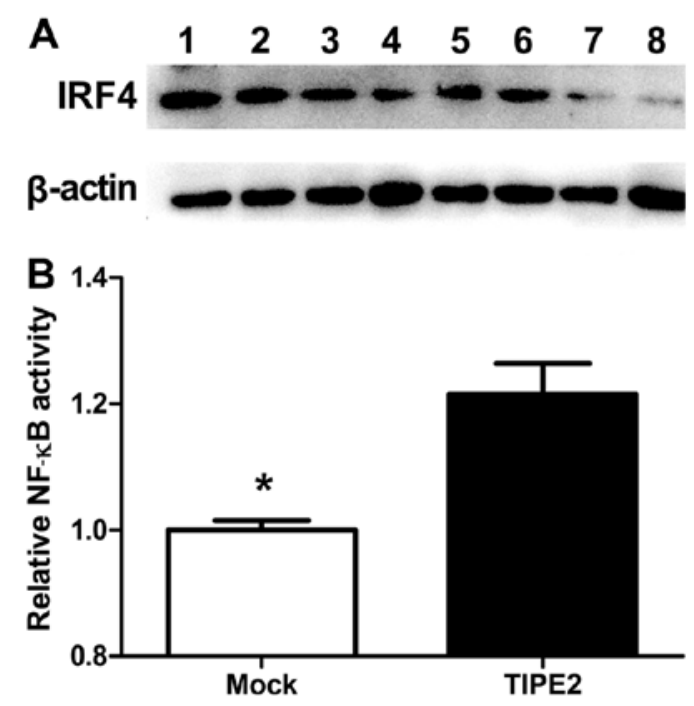

Figure 6. NF-кB pathway was employed by TIPE2 to regulate IRF4 expression. (A) The small molecule inhibitors: 1, control; 2, SB203580 (p38 inhibitor, $10 \mu \mathrm{M}$ ); 3, PD98059 (MEK inhibitor, $25 \mu \mathrm{M}$ ); 4, SP600125 (JNK inhibitor, $10 \mu \mathrm{M}) ; 5$, LY294002 (PI3K inhibitor, $40 \mu \mathrm{M}$ ); 6, SB431542 (TGF 3 inhibitor,

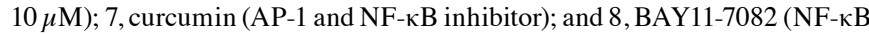
inhibitor, $4 \mu \mathrm{M}$ ) were employed for intervention in potential TIPE2-induced pathways involving IRF4 expression. TIPE2-induced IRF4 expression in AGS cells was significantly suppressed upon treatment with the NF- $\kappa B$ signaling pathway inhibitors BAY11-7082 and curcumin. (B) Variations in NF- $\kappa B$-dependent transcription in AGS cells were assessed with the NF- $\kappa B$ luciferase reporter assay, our results showed that NF- $\mathrm{KB}$ activity is increased in AGS cells.

and NF- $\kappa \mathrm{B}$ inhibitors), BAY11-7082 (NF- $\kappa \mathrm{B}$ inhibitor, $4 \mu \mathrm{M}$ ) and SB431542 (TGF $\beta$ inhibitor, $10 \mu \mathrm{M}$ ) were employed for intervention in potential TIPE2-induced pathways involving IRF4 expression. Notably, TIPE2-induced IRF4 expression in AGS cells was significantly suppressed upon treatment with BAY11-7082 and curcumin, whereas no obvious effects were observed with the other inhibitors (Fig. 6A). These results are consistent with previous finding that IRF4 expression is positively regulated by the NF- $\mathrm{KB}$ pathway. Variations in $\mathrm{NF}-\kappa \mathrm{B}$-dependent transcription in AGS cells were further assessed with the NF- $\mathrm{NB}$ luciferase reporter assay (Fig. 6B). Our results showed that NF- $\mathrm{KB}$ activity increased in AGS cells in response to changes in TIPE2 expression.

These findings collectively indicate that in AGS cells, activation of the NF- $\kappa B$ pathway is involved in TIPE2-induced IRF4 expression. Although the precise pathway responsible for maintaining NF- $\mathrm{\kappa B}$ activity upon TIPE2 expression remains unknown, we propose that restoration of TIPE2 activity may contribute, at least in part, to the initiation of IRF4 expression.

\section{Discussion}

To reduce mortality and improve the effectiveness of therapy, numerous studies have tried to find key biomarkers. Biomarkers are important molecular signposts of the biologic state of a cell at a specific condition, which play crucial roles in a number of processes important for tumor progression such as cell proliferation, motility, adhesion, invasion, survival and angiogenesis (21-25). TIPE2 induces cell death and inhibits tumor formation, providing a molecular bridge from inflammation to cancer. Zhang et al initially reported TIPE2 expression in several types of non-immune cells including the lung, stomach and liver (12). Separate studies in patients with systemic lupus erythematosus, chronic hepatitis B and chronic hepatitis $\mathrm{C}$ have shown significantly reduced levels of TIPE2 in peripheral blood mononuclear cells, compared to those in healthy individuals $(10,13,14,26)$. Our previous RT-qPCR and western blot results showed that TIPE2 upregulated p27 protein expression in AGS cells, this variation was verified in tumor tissues as well as by siRNA interference studies. Our present study disclosed variations in TIPE2 levels at different stages of gastritis progression and gastric cancer (GC) development, with a trend towards decreasing expression in the 
order of SG, AG, AH and GC. Based on these findings, we speculate that TIPE2 may be useful as a potential biomarker for GC progression, and therefore as a tool for the prevention of GC.

IRF4 is predominantly expressed in the immune system and plays an important role in its development and function $(27,28)$. IRF4 can function either as a transcriptional activator or repressor, depending upon its interactions with different transcription factors or DNA-binding domains on specific promoters (28). The enhanced understanding of the functional roles of IRF4 is dependent on the identification of genes that are uniquely regulated by the transcription factor. As IRF4 mRNA is induced upon TLR activation, IRF4 appears to participate in negative regulation of TLR signaling. IRF4 plays a significant role in disease progression and pathology under specific conditions. A recent study by Jo and Ren revealed that IRF4 functions as a tumor suppressor in bone marrow cells deficient in MyD88, an IRF4-interacting protein located in the cytoplasm (29). The tumor suppressor activity of IRF4 was lost in IRF association domain (IAD) deletion mutants, demonstrating that IRF4 suppresses BCR/ABL transformation through a novel cytoplasmic function involving its IAD domain (29). Additionally, Pathak et al (17) reported that c-Myc-induced leukemia is greatly accelerated in IRF4 heterozygous mutant mice, providing evidence that IRF4 functions as a classical tumor suppressor gene to inhibit c-Myc-induced leukemogenesis. The group further showed that deficiency of IRF4 accelerates loss of p27kip in EuMyc mice. Reconstitution of IRF4 in leukemic cells restored p27kip expression in leukemic cells and inhibited proliferation in vivo. Our experiments demonstrated that TIPE2 triggers a p27-associated signaling cascade that leads to restoration of control of the cell cycle and cell division. Association of IRF4 with p27 may therefore provide mechanistic links between IRF4 and cell proliferation inhibition.

NF- $\kappa B$ plays a key role in regulation of IRF4 expression. Previous studies have established that NF- $\kappa B$ is an upstream molecule of IRF4 that binds to its promoter and regulates expression. Wang et al reported that the IRF4 expression is initiated by $\mathrm{TNF} \alpha / \mathrm{NF}-\mathrm{\kappa B}$ signaling, suggesting that viral infection is inhibited through modulation of this pathway (30). Grumont and Gerondakis reported a novel mechanism in which Rel/NF- $\kappa \mathrm{B}$ represses the transcription of IFN-regulated genes in a cell type-specific manner (31). Moreover, Sharma et al (32) observed that IRF4 expression in HTLV-1-infected cells is driven through activation of NF- $\mathrm{KB}$ and NF-AT pathways. In the present study, expression of IRF4 was significantly decreased upon blockage of the NF- $\kappa B$ pathway. Furthermore, the NF- $\kappa B$ luciferase reporter assay revealed that NF- $\mathrm{kB}$ activity is slightly increased in AGS cells consistent with the results of Sharma et al (32).

In conclusion, TIPE2 acts as an inhibitor of GC cell growth and triggers an IRF4-associated signaling cascade that restores control of cell proliferation. Our study revealed a novel molecular mechanism by which TIPE2 regulates gastric cell proliferation. To the best of our knowledge, this is the first report of IRF4 as an inhibitor of epithelial cell proliferation and mediator of another negative TLR signaling regulator, TIPE2 to control cell growth.

\section{Acknowledgements}

We gratefully acknowledge the financial support from the Ph.D. Programs Foundation of Ministry of Education of China (20120131120046), The Fundamental Research Funds of Shandong University (2015JC010), the Natural Science Foundation (General programs 81272351), the Natural Science Foundation of Shandong Province (ZR2012HM020) and the Open Research Fund of State Key Laboratory of Environmental Chemistry and Ecotoxicology (KF2014-08).

\section{References}

1. Yang L, Parkin DM, Li LD, Chen YD and Bray F: Estimation and projection of the national profile of cancer mortality in China: 1991-2005. Br J Cancer 90: 2157-2166, 2004.

2. Yang L, Parkin DM, Ferlay J, Li L and Chen Y: Estimates of cancer incidence in China for 2000 and projections for 2005. Cancer Epidemiol Biomarkers Prev 14: 243-250, 2005.

3. Roder DM: The epidemiology of gastric cancer. Gastric Cancer 5 (Suppl 1): S5-S11, 2002

4. Fukata M and Abreu MT: Role of Toll-like receptors in gastrointestinal malignancies. Oncogene 27: 234-243, 2008.

5. Whitehead R, Truelove SC and Gear MW: The histological diagnosis of chronic gastritis in fibreoptic gastroscope biopsy specimens. J Clin Pathol 25: 1-11, 1972.

6. Coussens LM and Werb Z: Inflammation and cancer. Nature 420: 860-867, 2002

7. Liew FY, Xu D, Brint EK and O'Neill LA: Negative regulation of toll-like receptor-mediated immune responses. Nat Rev Immunol 5: 446-458, 2005.

8. Kondo T, Kawai T and Akira S: Dissecting negative regulation of Toll-like receptor signaling. Trends Immunol 33: 449-458, 2012.

9. Sun H, Gong S, Carmody RJ, Hilliard A, Li L, Sun J, Kong L, $\mathrm{Xu} \mathrm{L}$, Hilliard B, Hu S, et al: TIPE2, a negative regulator of innate and adaptive immunity that maintains immune homeostasis. Cell 133: 415-426, 2008.

10. Li D, Song L, Fan Y, Li X, Li Y, Chen J, Zhu F, Guo C, Shi Y and Zhang L: Down-regulation of TIPE2 mRNA expression in peripheral blood mononuclear cells from patients with systemic lupus erythematosus. Clin Immunol 133: 422-427, 2009.

11. Zhang G, Hao C, Lou Y, Xi W, Wang X, Wang Y, Qu Z, Guo C, Chen Y and Zhang Y: Tissue-specific expression of TIPE2 provides insights into its function. Mol Immunol 47: 2435-2442, 2010.

12. Zhang L, Shi Y, Wang Y, Zhu F, Wang Q, Ma C, Chen YH and Zhang L: The unique expression profile of human TIPE2 suggests new functions beyond its role in immune regulation. Mol Immunol 48: 1209-1215, 2011.

13. Xi W, Hu Y, Liu Y, Zhang J, Wang L, Lou Y, Qu Z, Cui J, Zhang G, Liang $\mathrm{X}$, et al: Roles of TIPE2 in hepatitis B virus-induced hepatic inflammation in humans and mice. Mol Immunol 48: 1203-1208, 2011.

14. Kong L, Liu K, Zhang Y-Z, Jin M, Wu BR, Wang WZ, Li W, Nan YM and Chen YH: Downregulation of TIPE2 mRNA expression in peripheral blood mononuclear cells from patients with chronic hepatitis C. Hepatol Int 7: 844-849, 2013.

15. Zhao Q, Zhao M, Dong T, Zhou C, Peng Y, Zhou X, Fan B, Ma W, Han $M$ and Liu S: Tumor necrosis factor- $\alpha$-induced protein-8 like-2 (TIPE2) upregulates p27 to decrease gastic cancer cell proliferation. J Cell Biochem 116: 1121-1129, 2015.

16. Negishi H, Ohba Y, Yanai H, Takaoka A, Honma K, Yui K, Matsuyama T, Taniguchi T and Honda K: Negative regulation of Toll-like-receptor signaling by IRF-4. Proc Natl Acad Sci USA 102: 15989-15994, 2005.

17. Pathak S, Ma S, Trinh L, Eudy J, Wagner KU, Joshi SS and Lu R: IRF4 is a suppressor of c-Myc induced B cell leukemia. PLoS One 6: e22628, 2011.

18. Fanzo JC, Hu CM, Jang SY and Pernis AB: Regulation of lymphocyte apoptosis by interferon regulatory factor 4 (IRF-4). J Exp Med 197: 303-314, 2003.

19. Budwit-Novotny DA, McCarty KS, Cox EB, Soper JT, Mutch DG, Creasman WT, Flowers JL and McCarty KS Jr: Immunohistochemical analyses of estrogen receptor in endometrial adenocarcinoma using a monoclonal antibody. Cancer Res 46: 5419-5425, 1986. 
20. Wang H, Sun Y, Liu S, Yu H, Li W, Zeng J, Chen C and Jia J: Upregulation of progranulin by Helicobacter pylori in human gastric epithelial cells via p38MAPK and MEK1/2 signaling pathway: Role in epithelial cell proliferation and migration. FEMS Immunol Med Microbiol 63: 82-92, 2011.

21. Hartwell LH and Kastan MB: Cell cycle control and cancer. Science 266: 1821-1828, 1994.

22. Woodburn JR: The epidermal growth factor receptor and its inhibition in cancer therapy. Pharmacol Ther 82: 241-250, 1999.

23. Coussens LM, Fingleton B and Matrisian LM: Matrix metalloproteinase inhibitors and cancer: Trials and tribulations. Science 295: 2387-2392, 2002.

24. Ferrara N: Role of vascular endothelial growth factor in physiologic and pathologic angiogenesis: Therapeutic implications. Semin Oncol 29 (Suppl 16): S10-S14, 2002.

25. Ghobrial IM, Witzig TE and Adjei AA: Targeting apoptosis pathways in cancer therapy. CA Cancer J Clin 55: 178-194, 2005.

26. Gus-Brautbar Y, Johnson D, Zhang L, Sun H, Wang P, Zhang S, Zhang L and Chen YH: The anti-inflammatory TIPE2 is an inhibitor of the oncogenic Ras. Mol Cell 45: 610-618, 2012.
27. Lu R: Interferon regulatory factor 4 and 8 in B-cell development. Trends Immunol 29: 487-492, 2008.

28. Marecki S and Fenton MJ: The role of IRF-4 in transcriptional regulation. J Interferon Cytokine Res 22: 121-133, 2002.

29. Jo SH and Ren R: IRF-4 suppresses BCR/ABL transformation of myeloid cells in a DNA binding-independent manner. J Biol Chem 287: 1770-1778, 2012.

30. Wang WL, Liu W, Gong HY, Hong JR, Lin CC and Wu JL: Activation of cytokine expression occurs through the $\mathrm{TNF} \alpha /$ $\mathrm{NF}-\kappa \mathrm{B}-$ mediated pathway in birnavirus-infected cells. Fish Shellfish Immunol 31: 10-21, 2011.

31. Grumont RJ and Gerondakis S: Rel induces interferon regulatory factor $4(I R F-4)$ expression in lymphocytes: Modulation of interferon-regulated gene expression by rel/nuclear factor kappaB. J Exp Med 191: 1281-1292, 2000.

32. Sharma S, Mamane Y, Grandvaux N, Bartlett J, Petropoulos L, Lin R and Hiscott J: Activation and regulation of interferon regulatory factor 4 in HTLV type 1-infected T lymphocytes. AIDS Res Hum Retroviruses 16: 1613-1622, 2000. 